\title{
Adenocarcinoma of the Gastroesophageal Junction Masquerading as Mallory-Weiss Syndrome
}

\author{
Takeshi Okamoto ${ }^{a, b} \quad$ Katsuyuki Fukuda ${ }^{a}$ \\ aDepartment of Gastroenterology, St. Luke's International Hospital, Tokyo, Japan; \\ bepartment of Hepato-Biliary-Pancreatic Medicine, Cancer Institute Hospital of Japanese \\ Foundation for Cancer Research, Tokyo, Japan
}

\section{Keywords}

Case report · Endoscopy · Gastric cancer · Stomach · Upper gastrointestinal bleeding

\begin{abstract}
Mallory-Weiss syndrome (MWS) is a relatively uncommon cause of upper gastrointestinal bleeding. While most cases can be managed conservatively, frequent transfusions or endoscopic hemostasis may be required in some cases. A 74-year-old man presented with hematemesis triggered by coughing. He had a history of violent chronic cough due to chronic obstructive pulmonary disease and was placed on aspirin and warfarin after multiple percutaneous coronary interventions and aortic valve replacement. Emergency esophagogastroduodenoscopy (EGD) revealed a mucosal tear in the gastroesophageal junction (GEJ) consistent with MWS which was treated with hemoclips. No tumor was seen at the bleeding site on follow-up EGD. Hematemesis after coughing was repeatedly seen $2-5$ months after the initial episode. Twelve months after the initial episode, the patient was admitted for pneumonia, heart failure, and tarry stools. Emergent EGD revealed a 30-mm mass at the GEJ, diagnosed as poorly differentiated adenocarcinoma on biopsy. Computed tomography revealed multiple enlarged lymph nodes consistent with metastases. The patient died 1 week later due to unrelated acute respiratory distress syndrome. Endoscopists should be aware that cancer of the GEJ may masquerade as repeated episodes of MWS.
\end{abstract}




\section{Introduction}

First reported in 1929 by Mallory and Weiss [1], Mallory-Weiss syndrome (MWS) refers to bleeding due to lacerations of the gastroesophageal junction (GEJ). MWS is a relatively rare cause of hematemesis, accounting for $3-15 \%$ of nonvariceal upper gastrointestinal bleeding [2]. MWS can cause significant bleeding in 23\% of cases, leading to frequent transfusions, endoscopic intervention, or even death [2-4]. However, most cases can be treated conservatively, with endoscopic hemostasis usually required only for active bleeding [5].

While obstructive gastric cancer may cause vomiting which in turn causes MWS [4], there are no reports of gastric cancer itself masquerading as MWS. Herein, we present a case of adenocarcinoma of the GEJ which presented with recurrent bleeding from the site of malignancy.

\section{Case Report/Case Presentation}

A 74-year-old man presented with hematemesis. He recalled abnormal sweating after waking up with malaise before a temporary loss of consciousness. When he regained consciousness, he vomited over 2 cups of blood after an extremely violent episode of cough. He had a history of chronic cough due to chronic obstructive pulmonary disease and was placed on aspirin and warfarin after multiple percutaneous coronary interventions and aortic valve replacement. He had a 40 pack-year smoking history but denied ever having alcohol. He also had no known drug or food allergies. Family history was unremarkable. He also had no history of hepatitis virus infections or liver disease.

Upon presentation, the patient appeared to be in moderated distress. His eyes remained open, but altered mental status was noted. He had a low body temperature of $35.1^{\circ} \mathrm{C}$, but vital signs were otherwise normal: blood pressure of 132/80 $\mathrm{mm} \mathrm{Hg}$, heart rate of 78 beats per minute, respiratory rate of 18 breaths per minute, and saturation of $98 \%$ on room air. While some blood was observed in the patient's mouth, physical examination of the chest and abdomen was unremarkable.

Laboratory values were remarkable for a hemoglobin level of $7.9 \mathrm{~g} / \mathrm{dL}$, blood urea nitrogen of $42.2 \mathrm{mg} / \mathrm{dL}$, and an international normalized ratio of 2.35. Computed tomography with contrast showed circumferential, edematous thickening of the GEJ wall and slight lymphadenopathy suggestive of a localized inflammatory process. Esophagogastroduodenoscopy (EGD) revealed a visible vessel on the posterior wall of the gastric cardia (Fig. 1a) and an ulcer-like mucosal tear on the anterior gastric wall near the GEJ (Fig. 1b). Both lesions were believed to be consistent with MWS and were treated with hemoclips. Other findings included closed-type atrophic gastritis with xanthomas and fundic gland polyps, consistent with previous Helicobacter pylori infection.

The patient was treated with blood transfusions and intravenous proton pump inhibitors. No tumor was seen at the clipped sites on follow-up EGD the next day (Fig. 1c). Oral intake was resumed on the fourth day of admission. The hospital course was uneventful, and the patient was discharged 8 days after admission.

Hematemesis after coughing was repeatedly seen 2-5 months after the initial episode, each time treated with hemoclips. Additional iatrogenic lacerations were observed during the course of each EGD procedure, as the patient coughed and retched throughout each procedure. Regenerative granulation tissue was observed after 3 months (Fig. 2a) but had spontaneously regressed by the next month (Fig. 2b). Another depressed lesion was observed under a previously applied hemoclip in the fifth month (Fig. 2c, d). Despite some surface irregularity being suspected on narrow-band imaging, no biopsies were taken due to severe bleeding. Follow-up was suspended as cardiologists who did not wish to suspend anticoagulation in this high-risk cardiac patient suspected that endoscopic procedures were the main cause of continued bleeding.

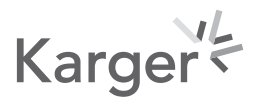




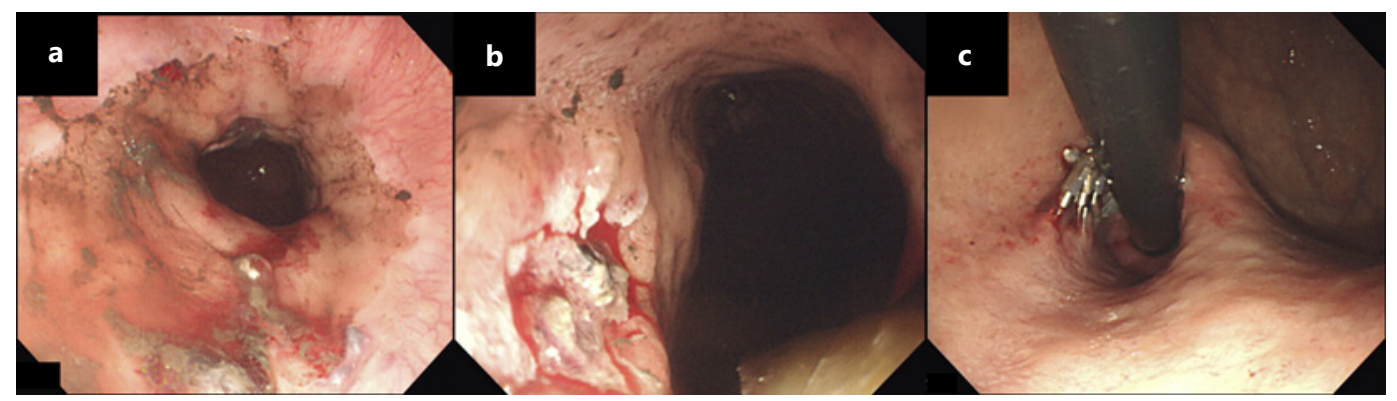

Fig. 1. EGD at initial presentation revealed a visible vessel on the posterior wall of the gastric cardia (a) and an ulcer-like mucosal tear on the anterior gastric wall near the GEJ (b). c No tumor was seen at the clipped sites on follow-up EGD the next day. EGD, esophagogastroduodenoscopy; GEJ, gastroesophageal junction.

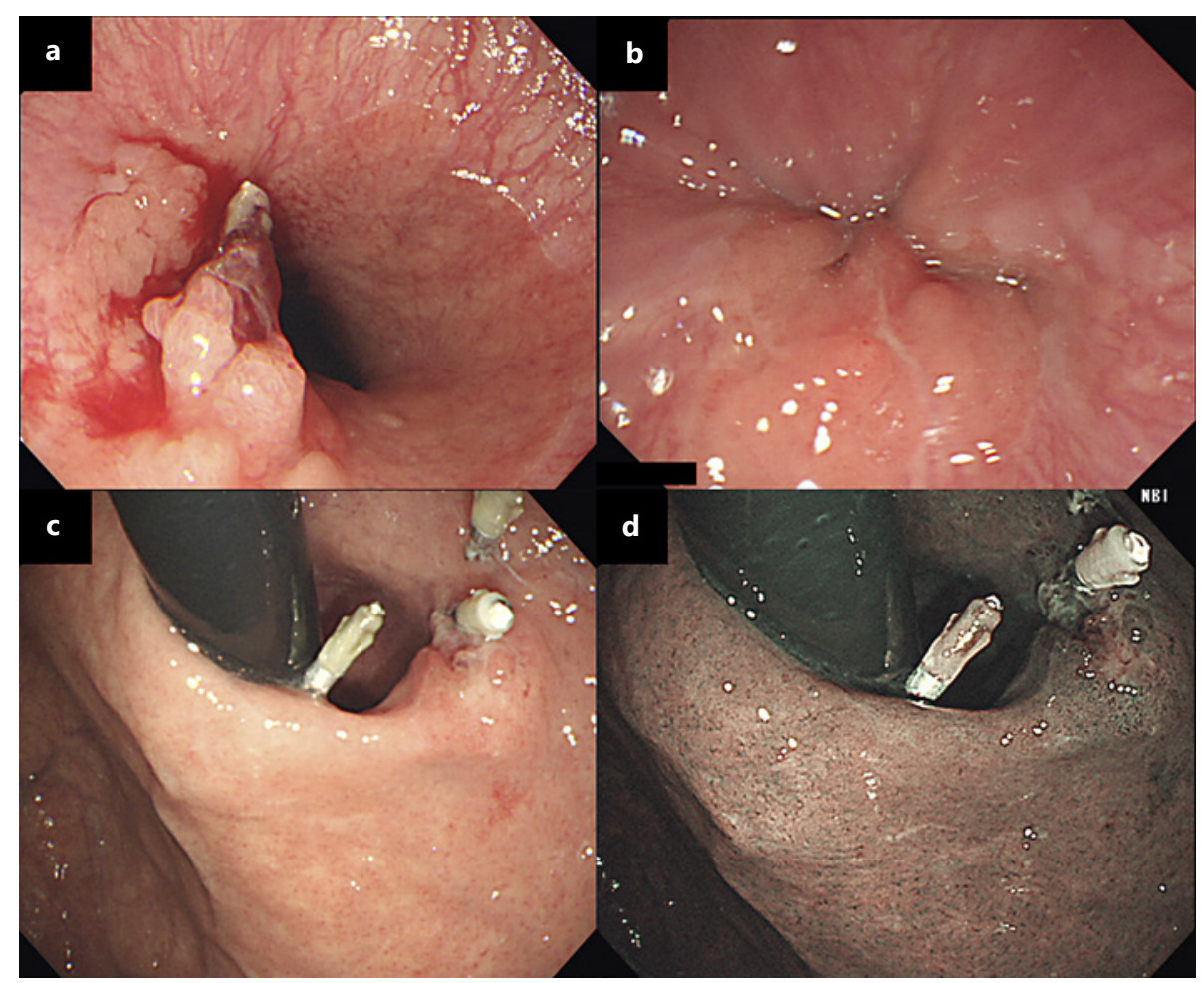

Fig. 2. a Regenerative granulation tissue was observed after 3 months. b The granulation tissue had spontaneously regressed by the fifth month. c White light and narrow-band imaging of another depressed lesion observed under a previously applied hemoclip in the fifth month (d).

Twelve months after the initial episode, the patient was admitted for pneumonia, heart failure, and tarry stools. Computed tomography with contrast revealed a large mass in the GEJ with multiple enlarged lymph nodes, and EGD revealed a 30-mm mass at the GEJ (Fig. 3a,b). Pathological evaluation showed proliferation of dysplastic cells with anisokaryosis and chromatin-rich nuclei adjacent to necrotic tissue and normal cells, consistent with poorly differentiated adenocarcinoma. The HER2 score was 0 . The patient was diagnosed with Siewert type 2 gastric cancer and multiple lymph nodes metastases. While his hemoglobin level was maintained after 4 units of red blood cell transfusions, the patient died 1 week later due to unrelated acute respiratory distress syndrome. 


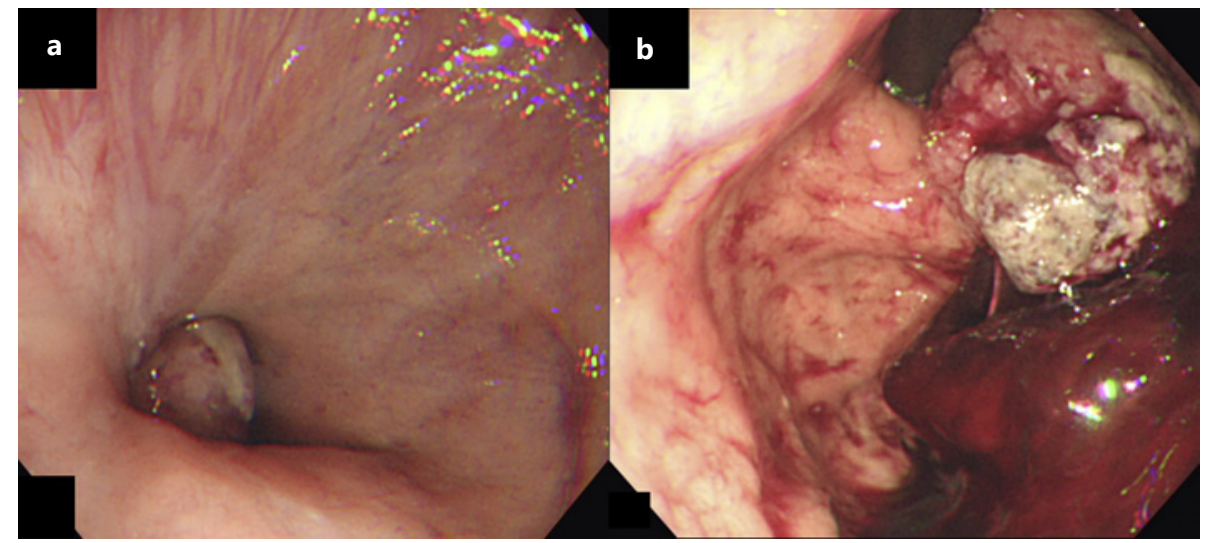

Fig. 3. Multiple masses with adherent blood clots were observed on antegrade (a) and retroflexed views of the gastric cardia (b).

\section{Discussion/Conclusion}

GEJ adenocarcinoma is a subtype of gastric adenocarcinoma which is believed to be a distinct entity from noncardiac gastric adenocarcinomas [6, 7]. It is often discovered in late stages and is associated with a poor prognosis. It can be difficult to diagnose due to the following reasons: (1) difficult visualization when the patient cannot inhale deeply to reduce intrathoracic pressure, (2) the subtle endoscopic findings, and (3) regenerative epithelial cells and inflammatory mesenchymal cells which may mimic carcinoma [6]. Reports on the association between GEJ cancer and H. pylori are mixed [8-10]. While Barrett's esophagus and associated adenocarcinoma are known to occur most commonly on the right anterior wall of the GEJ [11], there are no reports of the circumferential distribution of GEJ adenocarcinoma which is not associated with Barrett's esophagus.

MWS is known to occur most frequently on the right wall of the gastric side of the GEJ due to uneven pressure distribution across the circumference [11,12]. However, 1 report found almost even distribution between the right and left walls [4]. Multiple lacerations can also occur. It is therefore imprudent to differentiate MWS from cancer based on location alone.

Most cases of MWS are mild and may even go unnoticed. Large studies on MWS are scarce, with only 8 studies with over 100 cases in our literature review since 2000 [4, 12-18]. A population-based study found 2,342 cases of MWS in 783,037 EGDs (0.3\%), of which 421 (18\%) has active bleeding [13]. Another study reported that 71 of 546 iatrogenic MWS (13\%) required endoscopic hemostatic procedures [14]. A study on 698 cases on African American and Hispanic MWS patients found surprisingly high hemostatic failure (11\%), rebleeding $(12 \%)$, and mortality (10\%) rates [15]. On the other hand, 5 studies including a total of 985 cases found active bleeding in 35\%, nonbleeding visible vessels in $8.1 \%$, hemostatic failure in $0.3 \%$, rebleeding in $2.5 \%$, and death in $1.8 \%$ of cases [4, 12, 16-18].

MWS most commonly results from vomiting after alcohol consumption, but no risk factor may be identified in up to $23 \%$ of cases [3]. It can also rarely be induced by cough or hiccups [19-21], as in our case. Malignancies were found in 7 of 128 cases in 1 study [4], all of which may have contributed to vomiting but were not the direct source of bleeding from the GEJ. We found no reports of GEJ cancer masquerading as MWS.

We had assumed prematurely that the severe coughs, gag reflex, multiple anticoagulants, and EGD interventions were the causes of the frequent recurrence. Gag reflex, bleeding, clots, and severe bleeding also precluded a clear visualization of the lesion and made us hesitate from 
taking biopsies. The ulcerated lesion near the endoclip in the retroflexed view of the cardia in the follow-up after 5 months would usually lead us to suspect malignancy. However, tumor-like masses had arisen near the GEJ after applying hemoclips and had resolved spontaneously, leading us to suspect a benign, regenerative process. In addition, follow-up was suspended based on the cardiologists' request. Although studies on tissue scarring and granulation after endoscopic clipping are scarce, 1 study on colonic endoscopic mucosal resections found that clips gave rise to either mucosal elevations with normal pit patterns or cliprelated granulation tissue in $26 \%$ of cases, and can occur with or without residual clips [22]. A combination of these pitfalls and the jumping to conclusions bias led to the delayed diagnosis in this case.

In conclusion, we report a case of GEJ adenocarcinoma presenting as repeated episodes of MWS. A high index of suspicion for cancer may be warranted when repeated episodes of MWS occur.

\section{Acknowledgement}

This manuscript does not include any nonauthor contributors to acknowledge.

\section{Statement of Ethics}

The patient has given his written informed consent to publish this case (including publication of images). The identity of the patient has been protected. This case report complies with the guidelines for human studies and was conducted ethically in accordance with the World Medical Association Declaration of Helsinki. This study protocol was reviewed, and the need for approval was waived by the Ethics Committee at St. Luke's International Hospital.

\section{Conflict of Interest Statement}

The authors have no conflicts of interest to declare.

\section{Funding Sources}

This manuscript did not receive any funding.

\section{Author Contributions}

Takeshi Okamoto wrote the manuscript. Katsuyuki Fukuda critically reviewed the manuscript. Both authors gave final approval of the manuscript.

\section{Data Availability Statement}

All data generated or analyzed during this study are included in this article. Further inquiries can be directed to the corresponding author.

\section{Karger'}




\section{References}

1 Mallory GK, Weiss S. Hemorrhages from lacerations of the cardiac orfice of the stomach due to vomiting. Am J Med Sci. 1923;178:506-15.

2 Church NI, Palmer KR. Ulcers and nonvariceal bleeding. Endoscopy. 2003 Jan;35(1):22-6.

3 Kortas DY, Haas LS, Simpson WG, Nickl NJ 3rd, Gates LK Jr. Mallory-Weiss tear: predisposing factors and predictors of a complicated course. Am J Gastroenterol. 2001 Oct;96(10):2863-5.

4 He L, Li ZB, Zhu HD, Wu XL, Tian DA, Li PY. The prediction value of scoring systems in Mallory-Weiss syndrome patients. Medicine. 2019 May;98(22):e15751.

5 Chung IK, Kim EJ, Hwang KY, Kim IH, Kim HS, Park SH, et al. Evaluation of endoscopic hemostasis in upper gastrointestinal bleeding related to Mallory-Weiss syndrome. Endoscopy. 2002 Jun;34(6):474-9.

6 Abdi E, Latifi-Navid S, Zahri S, Yazdanbod A, Pourfarzi F. Risk factors predisposing to cardia gastric adenocarcinoma: insights and new perspectives. Cancer Med. 2019 Oct;8(13):6114-26.

7 Wang LD, Zheng S, Zheng ZY, Casson AG. Primary adenocarcinomas of lower esophagus, esophagogastric junction and gastric cardia: in special reference to China. World J Gastroenterol. 2003 Jun;9(6):1156-64.

8 Watari J, Chen N, Amenta PS, Fukui H, Oshima T, Tomita T, et al. Helicobacter pylori associated chronic gastritis, clinical syndromes, precancerous lesions, and pathogenesis of gastric cancer development. World J Gastroenterol. 2014 May 14;20(18):5461-73.

9 Helicobacter and Cancer Collaborative Group. Gastric cancer and helicobacter pylori: a combined analysis of 12 case control studies nested within prospective cohorts. Gut. 2001 Sep;49(3):347-53.

10 Pourfarzi F, Whelan A, Kaldor J, Malekzadeh R. The role of diet and other environmental factors in the causation of gastric cancer in Iran: a population based study. Int J Cancer. 2009 Oct 15;125(8):1953-60.

11 Kinoshita Y, Furuta K, Adachi K, Amano Y. Asymmetrical circumferential distribution of esophagogastric junctional lesions: anatomical and physiological considerations. J Gastroenterol. 2009;44(8):812-8.

12 Okada M, Ishimura N, Shimura S, Mikami H, Okimoto E, Aimi M, et al. Circumferential distribution and location of Mallory-Weiss tears: recent trends. Endosc Int Open. 2015 Oct;3(5):E418-24.

13 Corral JE, Keihanian T, Kröner PT, Dauer R, Lukens FJ, Sussman DA. Mallory Weiss syndrome is not associated with hiatal hernia: a matched case-control study. Scand J Gastroenterol. 2017 Apr;52(4):462-4.

14 Na S, Ahn JY, Jung KW, Lee JH, Kim DH, Choi KD, et al. Risk factors for an iatrogenic Mallory-Weiss tear requiring bleeding control during a screening upper endoscopy. Gastroenterol Res Pract. 2017;2017: 5454791.

15 Akhtar AJ, Padda MS. Natural history of Mallory-Weiss tear in African American and Hispanic patients. J Natl Med Assoc. 2011 May;103(5):412-5.

16 Ljubičić N, Budimir I, Pavić T, Bišćanin A, Puljiz Z, Bratanić A, et al. Mortality in high-risk patients with bleeding Mallory-Weiss syndrome is similar to that of peptic ulcer bleeding. Results of a prospective database study. Scand J Gastroenterol. 2014 Apr;49(4):458-64.

17 Lecleire S, Antonietti M, Iwanicki-Caron I, Duclos A, Ramirez S, Ben-Soussan E, et al. Endoscopic band ligation could decrease recurrent bleeding in Mallory-Weiss syndrome as compared to haemostasis by hemoclips plus epinephrine. Aliment Pharmacol Ther. 2009 Aug 15;30(4):399-405.

18 Lee S, Ahn JY, Jung HY, Jung KW, Lee JH, Kim DH, et al. Effective endoscopic treatment of Mallory-Weiss syndrome using Glasgow-Blatchford score and Forrest classification. J Dig Dis. 2016 Oct;17(10):676-84.

19 Annunziata GM, Gunasekaran TS, Berman JH, Kraut JR. Cough-induced Mallory-Weiss tear in a child. Clin Pediatr. 1996 Aug;35(8):417-9.

20 Dwivedi S, Al-Hamid H, Warren BJ. Mallory-Weiss tear after violent hiccups: a rare association. J Community Hosp Intern Med Perspect. 2017 Mar 31;7(1):37-9.

21 Guo F, Wang J, Liu K, Zeng Z, Luo F. Small-cell lung cancer with Mallory-Weiss syndrome as the prominent manifestation. Int J Clin Exp Pathol. 2019 Jul 1;12(7):2758-62.

22 Pellisé M, Desomer L, Burgess NG, Williams SJ, Sonson R, McLeod D, et al. The influence of clips on scars after EMR: clip artifact. Gastrointest Endosc. 2016 Mar;83(3):608-16. 